\title{
Educação Nutricional: uma Lacuna na Formação Médica
}

\section{Nutritional Education: a Gap in Medical Training}

Denise Bitar Vasconcelos Villacorta ${ }^{I}$ iD Charles Alberto Villacorta de Barros ${ }^{I}$ (D) Bernardo Felipe Santana de Macedo ${ }^{I}$ (iD Milena Coelho Fernandes Caldato ${ }^{I}$ iD

\section{PALAVRAS-CHAVE}

- Educação Médica.

-Terapia Nutricional.

- Currículo.

- Educação.

- Escolas Médicas.

- Nutrição.

Introdução: $O$ papel da nutrição na medicina tem sido modificado de uma função passiva, de uma terapia adjuvante, para uma terapia proativa e sofisticada, que previne diversos agravos à saúde e modifica a história natural da doença. Estudos recentes observam que a educação médica não contempla, de maneira suficiente e eficiente, os aspectos nutricionais do cuidado destinado ao paciente, formando médicos pouco confiantes em promover cuidados nutricionais a seus pacientes. Este estudo teve como objetivos analisar e descrever estudos científicos que tenham avaliado a educação nutricional nas escolas médicas, buscando, neste contexto, encontrar temas em nutrição importantes para a graduação em Medicina. Método: Esta pesquisa foi realizada por meio de uma scoping review (revisão de escopo), do tipo transversal, descritiva, após pesquisa de sinônimos utilizando as ferramentas Medical Subject Headings (MeSH) e Descritores em Ciências da Saúde (DeCS). Resultados: Encontraram-se inicialmente 1.057 publicações que passaram por triagens sequenciais até chegarmos a um total de 16 artigos que alcançavam o escopo desta pesquisa. A maioria dos artigos é dos Estados Unidos (50\%) $e$ avaliou de maneiras diferentes um total de 860 alunos de Medicina e 243 escolas médicas em relação ao ensino da nutrição. Conclusões: Nesta revisão, evidenciamos que, a despeito dos vários estudos que ratificam a relação bem estabelecida da nutrição na prevenção e no tratamento de doenças em nível ambulatorial ou hospitalar, a educação nutricional na formação médica não acompanhou essas evidências, e, por muitos anos, o assunto foi subestimado. No Brasil não foram encontrados estudos sobre esse tema com os descritores utilizados. 


\section{KEYWORDS}

- Medical Education.

- Nutritional Therapy.

- Curriculum.

- Education.

- Medical Schools.

- Nutrition.

\section{ABSTRACT}

Introduction: The role of nutrition in medicine has changed from a passive function, from an adjuvant therapy, to a proactive and sophisticated therapy that prevents various health problems and changes the natural history of the disease. Recent studies show up that medical education does not sufficiently and efficiently address the patient's nutritional aspects, thus training physicians who are not confident in providing nutritional care to their patients. This study aimed to analyze and describe scientific studies that have evaluated nutrition education in medical schools, seeking within this context to find nutrition topics important for undergraduate medical education. Methods: This research was conducted through a cross-sectional, descriptive, scoping review after searching for synonyms using MeSH (Medical Subject Headings) and DeCS (Health Sciences Descriptors) tools. Results: We initially found 1.057 publications that underwent sequential screening until reaching a total of 16 articles, which achieved the scope of this research. Most articles are from the United States of America (50\%), assessed a total of 860 medical students and 243 medical schools using different approaches regarding the teaching of nutrition. Final considerations: In this review, we have shown that despite several studies ratifying the well-established association between nutrition and prevention/treatment of diseases that require outpatient care, or at the hospital level, nutrition education in undergraduate medical school has not accompanied this evidence and, for many years, the subject has been underestimated. In Brazil, no studies were found on this topic with the used descriptors.

Recebido em 20/11/19

Aceito em 5/7/20

\section{INTRODUÇ̃̃O}

A nutrição humana envolve aspectos culturais, psicológicos, educacionais, sociais, políticos e econômicos. Recentemente, com o avanço nas tecnologias alimentares e na zootecnia, o cenário da alimentação se torna ainda mais complexo e, sem dúvida, gera impacto na saúde da população ${ }^{1}$.

Diversos autores reportaram o impacto que a alimentação tem no processo saúde-doença ${ }^{2,3-6}$. Pesquisa publicada em 2016 reportava a doença cardiovascular aterosclerótica como a principal causa de morte prevenível nos Estados Unidos (EUA) e que fatores alimentares comprovadamente reduzem a morbidade e mortalidade desse tipo de patologia ${ }^{7}$. Outras patologias crônicas como diabetes tipo 2 e hipertensão arterial também são fortemente influenciadas por práticas nutricionais ${ }^{8}$.

A desnutrição hospitalar tambémé ponto crítico. Pacientes desnutridos permanecem mais tempo internados e cursam com comprometimento de funções respiratórias e cardíacas, função imunológica diminuída e maiores taxas de morbidade e mortalidade ${ }^{9}$.

Em relação aos pacientes desnutridos internados em unidade de terapia intensiva (UTI), por exemplo, observam-se aumento no tempo de internação na unidade, ventilação mecânica prolongada e maiores taxas de mortalidade ${ }^{10}$. Porém, continua a ser um tema que não costuma fazer parte do currículo da graduação médica ${ }^{1,11}$.

Atualmente, a prevalência de desnutrição em ambiente hospitalar varia, entre os autores, de $20 \%$ a $60 \%{ }^{12,13}$. Evidências mostram que aproximadamente $50 \%$ dos pacientes já se encontram com algum grau de desnutrição no momento da internação, e, ao final desta, a avaliação nutricional realizada evidencia que essa taxa aumenta para $62 \%$,

Han et al. ${ }^{14}$ avaliaram o conhecimento e a percepção da nutrição em médicos residentes na atenção primária e constataram que $86 \%$ não se sentiam adequadamente treinados para discutir nutrição com pacientes. Entretanto, a nutrição é indissociável de todas as outras formas terapêuticas, sejam clínicas ou cirúrgicas ${ }^{15}$. Apesar disso, os próprios médicos relatam que se sentem sem condições de promover cuidados nutricionais ${ }^{16,17}$.
Alguns médicos residentes reconhecem que, nesse aspecto, existe uma carência de treinamento em seus programas de residência ${ }^{18,19}$. Devries et al. ${ }^{18}$, em seu estudo, verificaram que $31 \%$ dos cardiologistas e $21 \%$ dos residentes de cardiologia não lembram de assuntos relacionados à nutrição terem sido ministrados nas suas graduações, e $90 \%$ apontam o mesmo fato durante a residência de cardiologia.

Entre os fatores apontados como as causas da fragilidade na formação médica, estão um currículo muito denso e extenso, a dificuldade de encontrar professores especialistas na área e que defendam a inclusão desses assuntos, assim como a falta de um consenso de quais assuntos nutricionais deveriam ser abordados ${ }^{16,20}$.

Em 1977, foi publicada pesquisa na qual somente $19 \%$ das escolas médicas dos Estados Unidos possuíam algum curso sobre nutrição. Uma pesquisa realizada em 1985 com 45 escolas médicas dos Estados Unidos demonstrou que $60 \%$ das escolas dedicavam menos de 20 horas para a educação nutricional e $20 \%$ destinavam menos de dez horas ao assunto ${ }^{21}$.

Além disso, observa-se que as escolas médicas que incluem algum conteúdo nutricional o fazem geralmente nos anos iniciais da graduação. A falta de aplicabilidade clínica do assunto tende a fazer com que os estudantes não tenham a percepção da importância do assunto ${ }^{22,23}$.

Essa falta de preparo em cuidados nutricionais é evidente no ambiente hospitalar ${ }^{5,6}$. Ferreira et al., ${ }^{24}$ em 2017, avaliaram o conhecimento de médicos de diferentes clínicas sobre terapia nutricional parenteral (TNP) e constataram que $50 \%$ deles não se sentiam preparados para trabalhar com esse tipo de terapia Além de ser o primeiro item da prescrição médica no hospital, a dieta é complexa, pois tem variações no que diz respeito à administração, à apresentação, à composição e ao fracionamento ${ }^{25}$.

Considerando que a hipótese mais aceita para a insegurança médica em prescrever a dieta é o ensino insuficiente desse assunto no currículo da graduação, este trabalho visa fazer a investigação sobre como está o ensino nutricional nas escolas médicas segundo a literatura. 


\section{METODOLOGIA}

Esta pesquisa foi realizada na Universidade do Estado do Pará, por meio de uma scoping review (revisão de escopo), do tipo transversal, descritiva, com amostra por conveniência, a partir da busca de estudos acessíveis, indexados e prontamente disponíveis nas seguintes bases de dados: PubMed (US National Library of Medicine/ National Institute of Health), Eric (Education Resources Information Center), Bireme (Centro Latino-Americano e do Caribe de informações em Ciências da Saúde) e Google Acadêmico, desde dez anos antes do dia 31 de julho de 2019, último dia de coleta de dados. Após pesquisa de sinônimos utilizando as ferramentas Medical Subject Headings (MeSH) e Descritores em Ciências da Saúde (DeCS), os descritores foram definidos. Para melhorar a acurácia da pesquisa de artigos relevantes, aplicaram-se os seguintes filtros: dez anos de publicação (2009-2019), humanos e idiomas inglês, português e espanhol.

Quanto às fórmulas utilizadas para a pesquisa e seus respectivos operadores boolianos, elas foram realizadas de três formas: "Nutrition" $A N D$ "Medical Education" OR "Medical School" AND "Curriculum" AND "Nutrition Science" AND "Nutrition Therapy", publicados nos últimos dez anos, envolvendo seres humanos, escritos em inglês, português e espanhol; "Nutrition" AND "Medical Education" OR "Medical School" AND "Curriculum" AND "Nutrition Science" AND "Nutrition Therapy", com filtro temporal de dez anos; e "Nutrição" E "Educação Médica” OU "Escolas Médicas" E "Currículo" E "Ciências Nutricionais" E “Terapia Nutricional”.

A partir da formação do pool de artigos alcançados com os descritores definidos pela pesquisa, selecionaram-se os artigos. Algumas palavras e/ou termos foram definidos como excludentes por desviarem-se do objetivo deste estudo, como: "medicina veterinária", "educação infantil", "nutrição na pré-escola" e "política na educação".

Excluíram-se da revisão estudos que não avaliaram a população de interesse ou não analisaram pelo menos um dos desfechos definidos.
Os desfechos definidos para esta pesquisa foram:

- Foco nas escolas médicas: avaliação do ensino da nutrição nas escolas médicas.

- Foco no aluno: percepção e/ou avaliação do aluno de Medicina em relação à educação nutricional.

- Temas relevantes: temas em educação nutricional mais frequentemente recomendados para fazerem parte do currículo de Medicina.

Análise estatística

Os dados coletados foram tabulados em planilhas do programa Microsof Excel, tratados por métodos de estatística descritiva a partir das seguintes informações colhidas dos estudos: desenho do estudo, amostra estudada, local do estudo, intervenções realizadas e desfechos anunciados.

\section{RESULTADOS}

O total de artigos encontrados nas bases de dados escolhidas foi de 1.057 trabalhos referenciados até 31 de julho de 2019

Referente à distribuição desses manuscritos por bases de dados, quando se aplicou a fórmula "Nutrition" AND "Medical Education" OR "Medical School" AND "Curriculum" AND "Nutrition Science" AND "Nutrition Therapy", foram coletados 1.012 manuscritos apenas na plataforma PubMed, sem achados nas demais. Quanto à fórmula "Nutrition" AND "Medical Education" OR "Medical School" AND "Curriculum" AND "Nutrition Science" AND "Nutrition Therapy", computaram-se 16 trabalhos na plataforma Bireme e 28 na plataforma Google Acadêmico, totalizando 44 manuscritos para essa operação. Por fim, quando se realizou a busca com a fórmula "Nutrição" E "Educação Médica" OU "Escolas Médicas" E "Currículo" E "Ciências Nutricionais" E "Terapia Nutricional", nenhum trabalho foi exibido em quaisquer bases de dados.

A síntese dessa seleção pode ser observada no fluxograma da Figura 1.

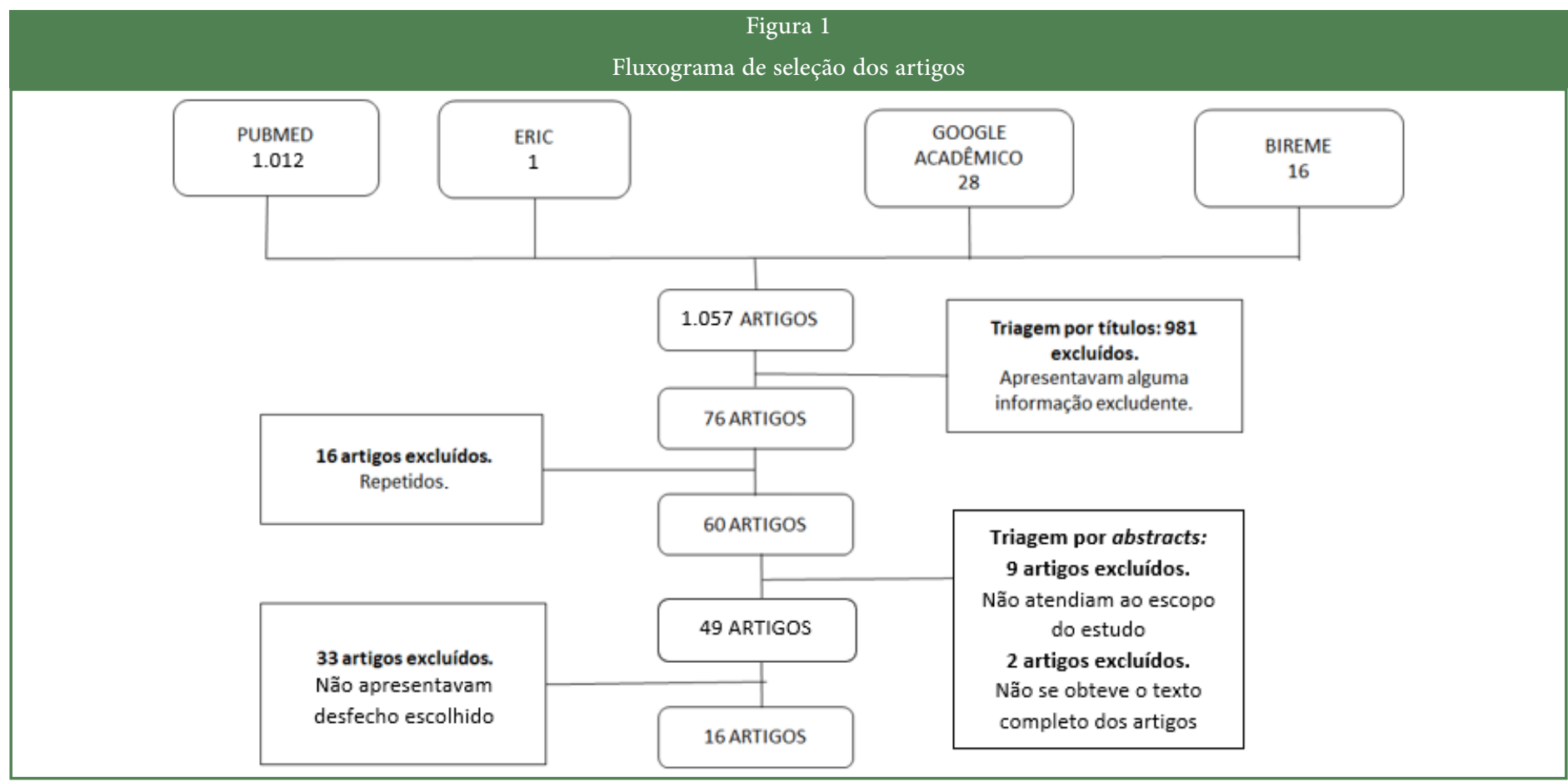

Fonte: Elaborada pelos autores. 


\section{Característica dos estudos incluídos}

A pesquisa resultou em estudos de sete países e três de caráter multicêntrico (um mundial, um europeu, um africano), com 50\% dos trabalhos (oito) realizados nos Estados Unidos.

Após o processo de triagem e leitura dos artigos completos, eles foram classificados quanto ao desenho de pesquisa apresentado. Do total de 16 artigos, sete $(43,75 \%)$ foram do tipo transversal, e o segundo tipo mais frequente de trabalho foi do método antes e depois (before and after), representado por três artigos (18,75\%) (Gráfico 1).

É importante ressaltar que alguns estudos apresentavam mais de um desfecho. Entre os desfechos definidos e analisados nos trabalhos que fizeram parte do escopo da pesquisa estão os seguintes:

- Desfecho 1: Encontrado em $31,25 \%$ dos estudos avaliados. Foco nas escolas médicas. Das 243 escolas médicas avaliadas, mais de 50\% apresentavam menos do que 25 horas de formação nutricional em seu currículo.

- Desfecho 2: Encontrado em 50\% dos estudos avaliados. Foco no aluno. Dos 860 alunos questionados nas pesquisas dos diferentes artigos, mais da metade referiu que não se sentia confiante em manejar assuntos relacionados à nutrição.

- Desfecho 3: Encontrado em $43,75 \%$ dos estudos avaliados. Temas relevantes. Entre os temas mais frequentemente sugeridos para inclusão no ensino médico, destacaram-se três grupos principais:

1. Temas sobre ciências nutricionais básicas, como estudo dos macro e micronutrientes, gasto energético, avaliação nutricional e risco nutricional.

2. Temas sobre orientações nutricionais aplicadas nas doenças crônicas, como cardiovasculares, obesidade, hipertensão, diabetes e câncer.

3. Temas sobre suporte nutricional no doente hospitalizado, incluindo nutrição enteral e parenteral.
Síntese dos estudos

A seleção dos 16 artigos utilizados por esta pesquisa, com as suas principais características, é apresentada no Tabela 1.

\section{DISCUSSÃO}

O papel da nutrição na medicina tem sido modificado de uma função passiva para uma terapia proativa e sofisticada, que previne diversos agravos à saúde e modifica a história natural da doença ${ }^{30}$. Mesmo assim, as causas mais prevalentes de morbidade e mortalidade em países desenvolvidos e em desenvolvimento estão relacionadas direta ou indiretamente à dieta ${ }^{2}$.

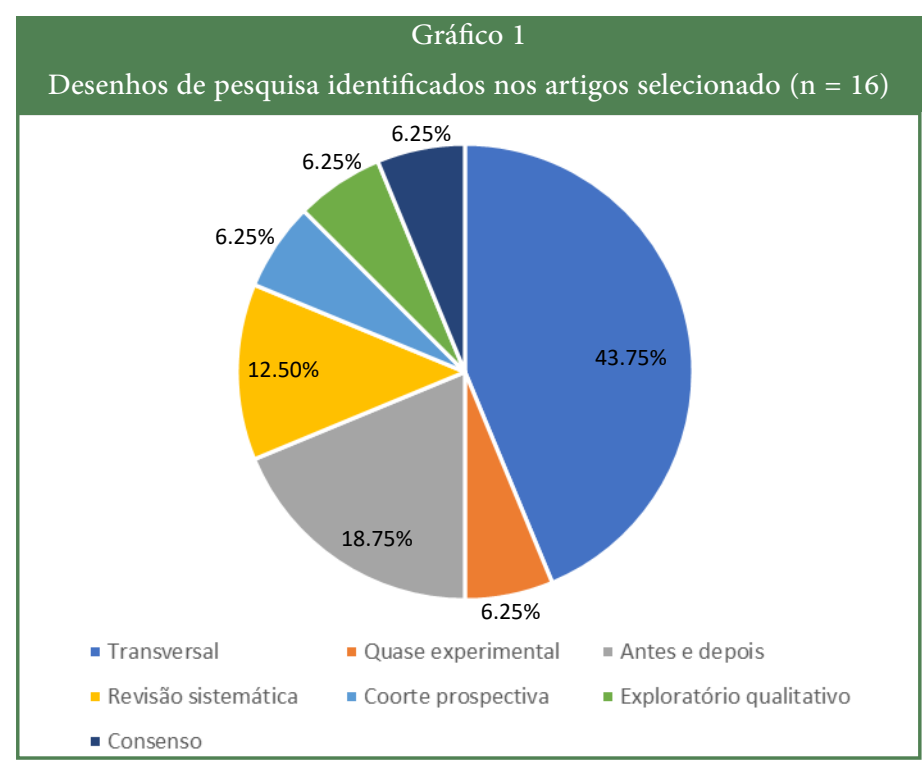

Fonte: Elaborado pelos autores.

\begin{tabular}{|c|c|c|c|c|c|c|}
\hline \multicolumn{7}{|c|}{ Tabela 1} \\
\hline $\begin{array}{l}\text { Autor/ano/local do } \\
\text { estudo }\end{array}$ & Amostra & $\begin{array}{l}\text { Tipo de } \\
\text { estudo }\end{array}$ & Intervenção & $\begin{array}{l}\text { Desfecho } 1 \text { - Nutrição nas } \\
\text { escolas médicas }\end{array}$ & Desfecho 2 - Foco no aluno & $\begin{array}{l}\text { Desfecho } 3 \text { - Temas } \\
\text { relevantes }\end{array}$ \\
\hline $\begin{array}{l}\text { Adams et al., } 2010 \\
\text { (Estados Unidos) }^{22}\end{array}$ & $\begin{array}{l}109 \text { escolas } \\
\text { médicas }\end{array}$ & $\begin{array}{l}\text { Trans- } \\
\text { versal }\end{array}$ & NA & $\begin{array}{l}\text { Média de horas sobre } \\
\text { nutrição: } 19,6 . \text { Das escolas } \\
\text { médicas, } 27 \%(28 / 105) \\
\text { forneciam o mínimo de } 25 \\
\text { horas. Trinta escolas realizam } \\
12 \text { horas ou menos de } \\
\text { instrução nutricional. }\end{array}$ & NA & NA \\
\hline $\begin{array}{l}\text { Walsh et al., } 2011 \\
\text { (Estados Unidos) }^{15}\end{array}$ & $\begin{array}{l}125 \text { alunos } \\
\text { de Medicina }\end{array}$ & $\begin{array}{l}\text { Quase } \\
\text { experi- } \\
\text { mental }\end{array}$ & $\begin{array}{l}\text { Currículo nutricional } \\
\text { dedicado versus integrado. } \\
\text { Curso dedicado à nutrição } \\
\text { com } 28 \text { horas, com } 14 \text { horas } \\
\text { semanais, } 45 \text { minutos de } \\
\text { aula, } 90 \text { minutos de atividade } \\
\text { versus introdução à nutrição } \\
\text { clínica com algumas aulas } \\
\text { e atividades, com demais } \\
\text { assuntos inseridos no estudo } \\
\text { de órgãos e sistemas. }\end{array}$ & $\mathrm{NA}$ & $\begin{array}{l}\text { Apesar de os alunos } \\
\text { preferirem o curso } \\
\text { dedicado à nutrição, em } \\
\text { ambas as metodologias } \\
\text { o aluno adquiriu } \\
\text { competências em nutrição } \\
\text { clínica semelhantes. }\end{array}$ & $\begin{array}{l}\text { Macro/micronutrientes, } \\
\text { obesidade, avaliação, } \\
\text { aconselhamento } \\
\text { nutricional, entre outros. }\end{array}$ \\
\hline
\end{tabular}




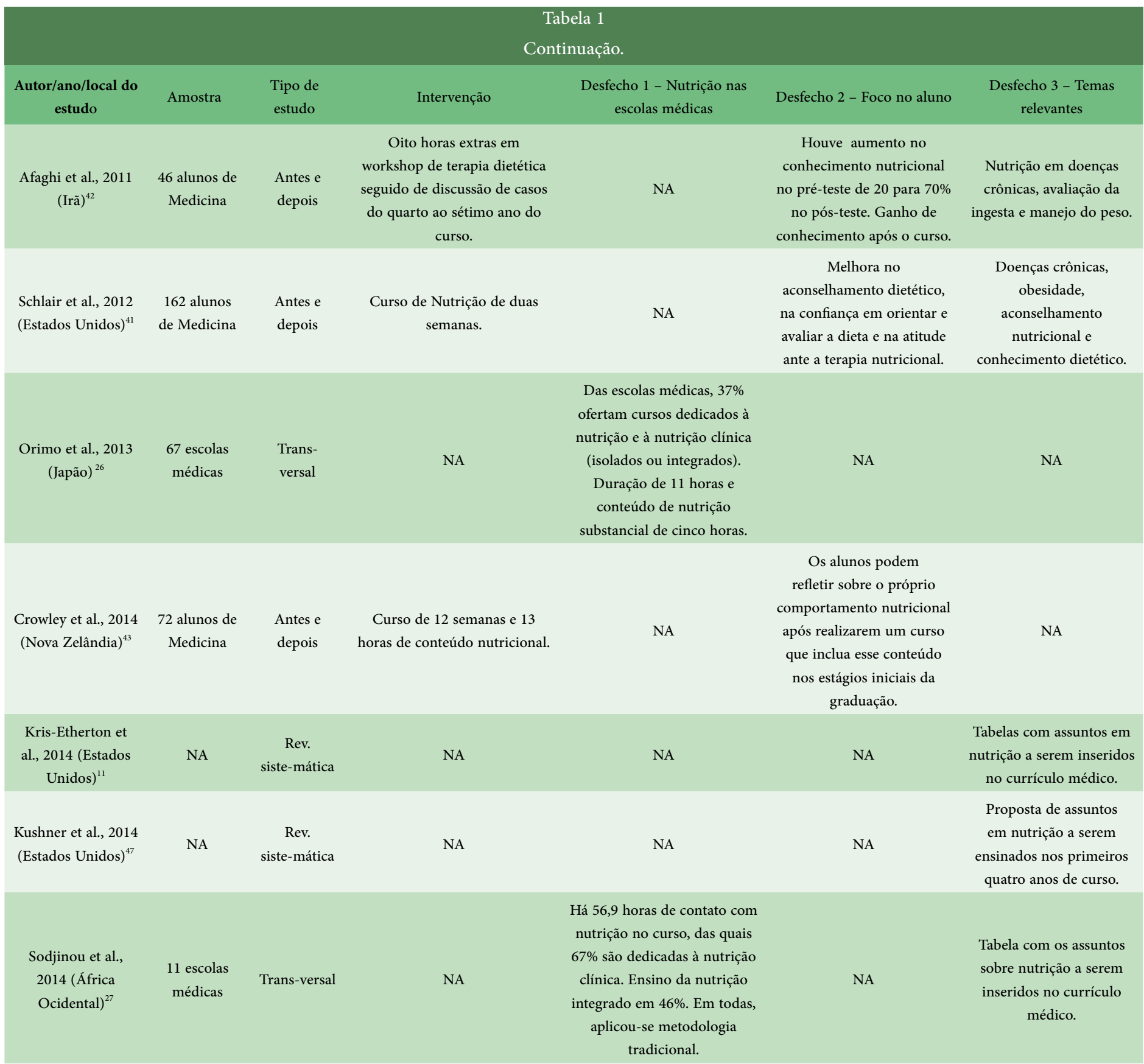

Dos alunos, $29 \%$

consideram suficiente a carga horária de nutrição clínica no curso de Medicina. Contato com nutrição clínica de 2,8 semanais, em média. Quatro ou mais semanas estão associadas a maior confiança no manejo da nutrição clínica.

Dificuldade de iniciar a nutrição clínica com a terapêutica farmacológica.

\begin{tabular}{|c|c|c|c|c|c|}
\hline $\begin{array}{l}\text { Frantz et al., } \\
2016 \text { (Estados } \\
\text { Unidos) }^{46}\end{array}$ & $\begin{array}{l}122 \text { alunos } \\
\text { de Medicina }\end{array}$ & $\begin{array}{l}\text { Trans- } \\
\text { versal }\end{array}$ & NA & NA & $\begin{array}{l}\text { semanais, em média. } \\
\text { Quatro ou mais semanas } \\
\text { estão associadas a maior } \\
\text { confiança no manejo } \\
\text { da nutrição clínica. } \\
\text { Dificuldade de iniciar a } \\
\text { nutrição clínica com a } \\
\text { terapêutica farmacológica. }\end{array}$ \\
\hline
\end{tabular}




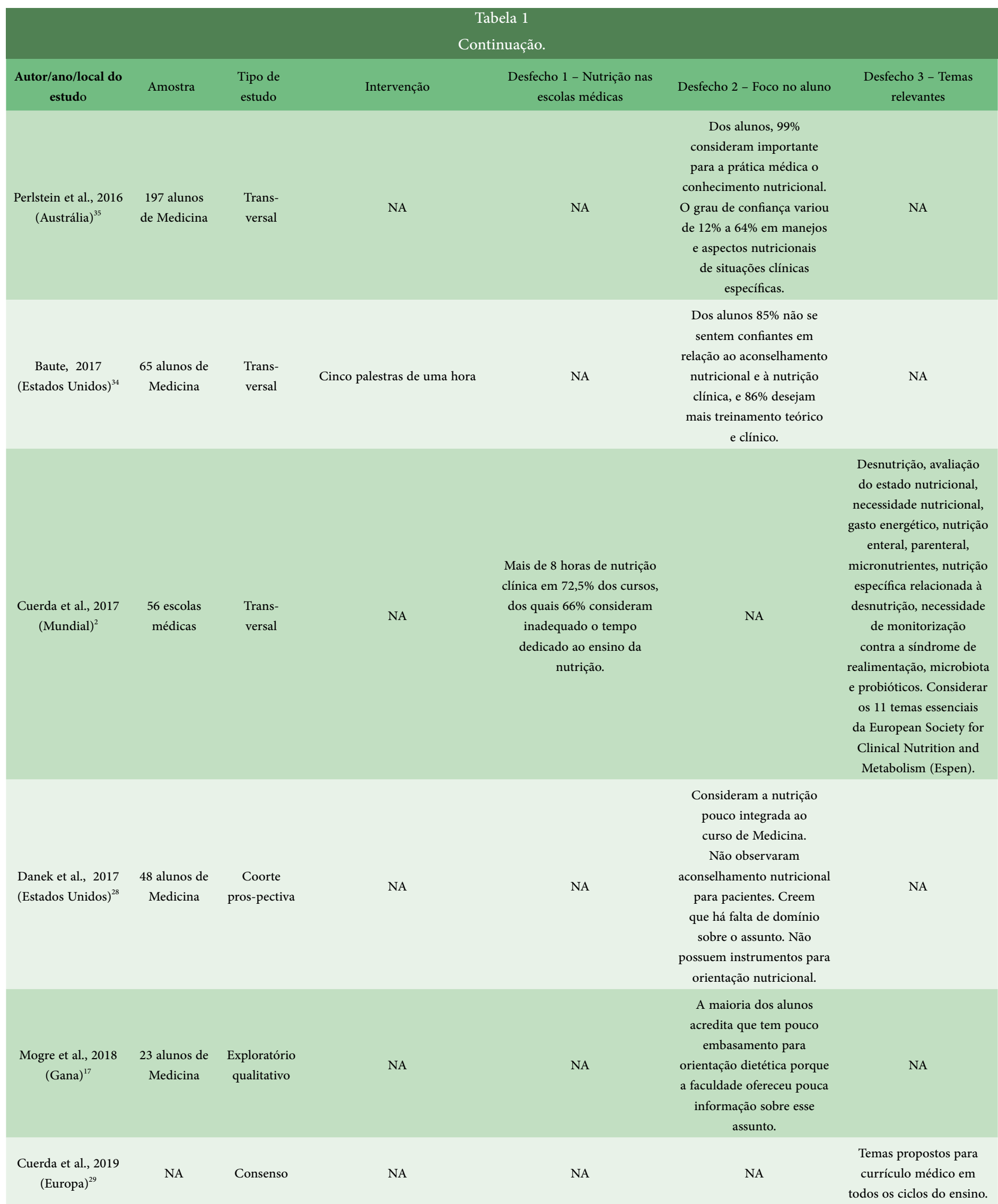

Fonte: Elaborado pelos autores.

NA: não se aplica; 
No ambiente hospitalar, o problema é latente. Um estudo nacional envolvendo quatro mil pacientes, publicado em 2001, demonstrou uma taxa de desnutrição hospitalar de 48\%. Passados mais de 15 anos, uma metanálise publicada em 2017, tendo como amostra 29.474 pacientes de 12 países da América Latina, dos quais 57,7\% eram brasileiros, evidenciou uma taxa de desnutrição hospitalar ainda maior, alcançando até $60 \%{ }^{31,32}$.

A educação de massa é entendida como o melhor caminho para modificar os hábitos nutricionais que cursam com o processo de doença. Esse fato se mostra claro na Lei $n^{\circ} 13.666 / 2018$ que torna obrigatória a educação alimentar e nutricional nos currículos escolares de alunos dos ensinos fundamental e médio de instituições públicas e privadas de todo o Brasil ${ }^{33}$.

Paradoxalmente, não há regulamentações em relação a quanto de conhecimento nutricional o médico em formação precisa obter para se tornar um agente modificador dessa realidade. $\mathrm{E}$ a falta de estudos publicados no Brasil sobre a educação nutricional nas escolas médicas sugere que nem foi iniciada a discussão em relação a essa fragilidade. Recentemente um estudo randomizado e controlado demonstrou o potencial impacto positivo em pacientes que participaram de curso de educação nutricional ministrado por estudantes de Medicina ${ }^{34}$.

Apesar da crescente importância dos aspectos nutricionais no manejo terapêutico do paciente e de o médico estar posicionado na linha de frente para conscientização de hábitos alimentares mais saudáveis, essa competência não tem sido alcançada pelas escolas formadoras de profissionais médicos de todo o mundo, tanto que um estudo global capitaneado pela European Society for Clinical Nutrition and Metabolism (Espen), com representação de 57 países, demonstrou que a educação nutricional nos cursos médicos é heterogênea e insuficiente ${ }^{2,11}$.

Uma pesquisa realizada na Austrália com alunos de Medicina constatou que $99 \%$ dos estudantes consideraram importante a educação nutricional e variou de $12 \%$ a $64 \%$ o grau de confiança referido por eles em manejar os cuidados nutricionais ${ }^{35}$.

A preocupação com o ensino nutricional nas escolas não é um fenômeno recente fora do Brasil. Em 1985, a Academia Nacional de Ciências (National Academy of Sciences - NAS), por meio de seu Comitê sobre Nutrição na Educação Médica (Committee on Nutrition in Medical Education), apresentou à sociedade o trabalho intitulado Nutrition education in U.S. medical schools (Educação nutricional nas escolas médicas dos Estados Unidos), considerado um marco na literatura médica sobre o tema ${ }^{36}$.

Impulsionado por esse estudo, foi lançado o Prêmio de Nutrição Acadêmica (Nutrition Academic Award - NAA) que promoveu a integração do conteúdo nutricional no currículo médico e produziu diretrizes de como realizar essa integração. Entretanto, em 2005 os recursos para esse programa foram suspensos, limitando seu impacto a apenas 21 escolas médicas norte-americanas. Passadas três décadas da avaliação nacional organizado pela NAS, a nutrição nas escolas médicas ainda é uma questão a ser resolvida ${ }^{21}$.

A relevância do tema para a saúde pública norte-americana é clara quando se considera que os principais agravos à saúde nos Estados Unidos são obesidade, doenças cardiovasculares, diabetes e câncer, os quais estão diretamente relacionadas com fatores alimentares e hábitos de vida potencialmente preveníveis ${ }^{9}$.

Enquanto os autores internacionais discutem, há mais de 30 anos, a deficiência na educação nutricional nas escolas médicas e nos programas de residência, propondo ações e conferências específicas para esse tema ${ }^{37}$, na literatura pesquisada, a partir da fórmula de descritores utilizada, não foi encontrado nenhum trabalho realizado no Brasil. Esse fato preocupa, visto que no Brasil levantamentos recentes evidenciaram que mais de $50 \%$ da população está acima do peso e que a prevalência de desnutrição hospitalar pode chegar a até $60 \%$ dos pacientes internados ${ }^{38,39}$.

Quaresma et al. ${ }^{40}$, em 2019, avaliaram médicos plantonistas de uma UTI de um hospital público no Brasil e constataram que $65 \%$ dos entrevistados possuem dificuldade de reconhecer pacientes em risco nutricional.

Entre as barreiras descritas para o avanço do ensino da nutrição nas escolas médicas, estão a baixa aplicabilidade clínica, a percepção de que o cuidado nutricional não é responsabilidade do médico, a falta de conhecimento da faculdade em nutrição clínica, a desmotivação dos professores em lecionar o assunto, o currículo superlotado, entre outros ${ }^{17}$.

$\mathrm{Na}$ última década (2009-2019), foco desta pesquisa, verificou-se que $43,75 \%$ dos estudos selecionados foram do tipo transversal. Isso sugere que os gestores da educação médica buscam aumentar a discussão sobre o tema trabalhando inicialmente para definir um diagnóstico situacional e assim conduzir o processo a ações mais contundentes.

Outros trabalhos foram do tipo antes e depois, $18,75 \%$, tendo como intervenções cursos dedicados à nutrição clínica que não faziam parte do currículo regular da graduação de Medicina. É perfeitamente inteligível que uma mudança curricular não é simples de se realizar, pois turmas mais avançadas, por exemplo, não se beneficiariam das inserções curriculares somente em semestres iniciais do curso.

Apesar de antiga, a recomendação mínima de 25 horas de ensino de nutrição para o currículo médico é ponto de partida e referencial para a maioria das intervenções. Foram descritos cursos de nutrição nos seguintes formatos: apenas cinco palestras; outros com carga horária de 13 horas; duas semanas de duração e ainda aqueles que são inseridos por três anos no ciclo clínico da graduação ${ }^{34,41-43}$.

Quanto à população avaliada em cada estudo selecionado, duas foram as encontradas: a faculdade e o aluno. As escolas médicas foram avaliadas por meio de questionários encaminhados aos coordenadores $\mathrm{e}$ gestores das instituições, o que pode configurar um viés de seleção, pois o responsável por responder ao formulário era o maior interessado em oferecer uma fotografia mais "adequada" do que a realidade era de fato.

Das 243 escolas avaliadas nos diversos estudos somados, em 40 não foi possível identificar quantas horas de educação nutricional foram ministradas. Nas escolas em que se pôde avaliar a carga horária, constatou-se que mais de $50 \%$ ofereciam menos do que o mínimo de 25 horas preconizado pela $\mathrm{NAS}^{36}$, variando de 4,2 a 19,6 horas. Quando se considera que um curso de Medicina possui carga horária mínima de 7.200 horas, fica evidente que o tempo para o ensino da nutrição é pouco valorizado.

Nas Diretrizes Curriculares Nacionais (DCN) do curso de Medicina, publicadas em 2014, não há praticamente nenhuma linha sobre os cuidados nutricionais do paciente. $\mathrm{Na}$ subseção "Da atenção às necessidades individuais de saúde", o artigo 13, que trata dos planos terapêuticos, cita como um dos objetivos o "estabelecimento, a partir do raciocínio clínico-epidemiológico em contextos específicos, de planos terapêuticos, contemplando as dimensões de promoção, prevenção, tratamento e reabilitação". Nesse contexto, planejar a intervenção terapêutica significa estar preparado para todos os itens como o cuidado com a alimentação, pois este pode se configurar tanto como prevenção

REVISTA BRASILEIRA DE EDUCAÇC̃̃ MÉDICA

7 44 (4) : e107; 2020 
quanto como tratamento de algumas patologias ${ }^{44}$.

Recentemente, Neumann et al. ${ }^{45}$ adaptaram e aprimoraram para a língua portuguesa as competências para alunos do internato médico, com base na avaliação de 15 Entrustable Professional Activities (EPAs). Destacamos duas EPAs que se relacionam fortemente ao ensino nutricional: colaborar como membro de uma equipe multiprofissional e educar pacientes: manejo da doença, promoção da saúde e medicina preventiva.

A avaliação do estudante de Medicina foi semelhante à das escolas médicas, em que se utilizaram questionários de percepção e, às vezes, de conhecimento. Os acadêmicos eram selecionados e avaliados por pesquisadores da própria instituição de ensino, o que favorecia o viés de avaliação.

Dos 860 alunos questionados nos estudos selecionados, mais de $50 \%$ mencionaram que não se sentiam preparados para o manejo dos cuidados nutricionais destinados ao paciente. Frantz et al. ${ }^{46}$ conduziram uma pesquisa com 122 internos do curso de Medicina, dos quais $71 \%$ referiram que se sentiam inadequadamente preparados para praticar nutrição clínica.

$\mathrm{Na}$ tentativa de resolver essa deficiência, alguns cursos introduziram a nutrição a partir de temas básicos, como estudo de macro e micronutrientes, gasto energético e necessidades nutricionais, nos dois primeiros anos da graduação, chamados de pré-clínicos ${ }^{42}$. Porém, com o surgimento de evidências de que doenças crônicas, como as cardiovasculares, a diabetes, a hipertensão e o câncer, sofrem influência direta da alimentação e que, portanto, para o tratamento delas, era necessária a intervenção médica, começou-se a inserir o assunto nos quatro primeiros anos de graduação, com o enfoque mais clínico no terceiro e quarto ano, buscando desenvolvimento de habilidades no manejo nutricional dos pacientes e se utilizando de métodos de aprendizado baseado em problemas ${ }^{47}$.

Nesta pesquisa, $50 \%$ dos artigos apresentaram o desfecho 3, relacionado aos temas mais frequentemente citados e considerados mais relevantes para fazerem parte do currículo médico. Os assuntos foram reunidos em três grupos.

Os temas de nutrição básica foram citados por todos os artigos, visto que esse conhecimento é elementar para o entendimento da fisiopatologia dos distúrbios nutricionais e para a viabilização da aplicabilidade clínica. Entre eles, destaca-se o conhecimento sobre macro e micronutrientes, necessidades nutricionais e avaliação nutricional e do gasto energético. Dados do Sistema de Vigilância de Fatores de Risco e Proteção para Doenças Crônicas (Vigitel) do Ministério da Saúde publicados em 2017 evidenciaram que somente $24,9 \%$ a $49,8 \%$ dos brasileiros fazem consumo de frutas e hortaliças regularmente. Essa informação sugere a precária conscientização da população quanto às principais fontes saudáveis de macro e micronutrientes ${ }^{48}$.

O manejo nutricional das doenças crônicas influenciadas pela dieta também foi tema frequentemente citado. São exemplos: as doenças cardiovasculares, que configuram uma das principais causas de morte no mundo; a hipertensão, diretamente relacionada ao consumo de sal e alimentos embutidos; a diabetes, que tem como pilar de seu controle um adequado consumo de carboidratos; alguns tipos de câncer, que podem ter seu gatilho a partir de fatores oncogênicos presentes na alimentação; e, por fim, a obesidade, que tem influência direta do padrão alimentar da população ${ }^{17,49,50}$.

Por fim, um tema que tomou maiores proporções na década de 1970, quando surgiram as primeiras publicações sobre desnutrição hospitalar, e segue em ascensão até os dias atuais foi a terapia nutricional no paciente hospitalizado. Esse tema merece destaque porque envolve o cuidado do doente em situações patológicas limítrofes. E diferentemente de outros ambientes de prática, o médico é o disparador do cuidado nutricional por meio de sua indicação e prescrição. Exige um conhecimento diferenciado, visto que os tipos de terapia nutricional, como a nutrição enteral e parenteral, além de representarem um custo significativamente maior para o hospital, são permeados por maiores riscos e possibilidades de complicações no processo de execução.

Para a regulação do uso desse tipo de terapia, foram criadas, no Brasil, pelo Ministério da Saúde normativas que regulamentam todo o processo de uso de nutrição enteral e parenteral, desde sua indicação até sua administração, passando por controles de qualidade, do transporte e da manipulação ${ }^{51,52}$.

\section{CONSIDERAÇÕES FINAIS}

Nesta revisão de escopo, evidenciamos que, a despeito dos vários estudos ratificando a relação bem estabelecida da nutrição, tanto na prevenção e no tratamento de doenças crônicas que demandam cuidados ambulatoriais, quanto em patologias que dependem de terapias em nível hospitalar, a educação nutricional na formação médica não acompanhou essas evidências, e, por muitos anos, o assunto foi subestimado. Nos dias atuais, ele vem sendo cada vez mais discutido fora do Brasil.

A ausência de estudos brasileiros nessa área oportuniza o desenvolvimento de novas pesquisas no sentido de diagnóstico da realidade nacional e posterior busca de estratégias condizentes com o contexto encontrado. No momento, assim como nos outros países, cursos extracurriculares podem ser uma alternativa para suprir o conhecimento e habilidades que não estejam sendo entregues pelas faculdades de Medicina.

\section{REFERÊNCIAS}

1. Godoy A, Lopes D, Garcia R. Transformações socioculturais da alimentação hospitalar. Hist Ciênc Saúde-Manguinhos. 2007;14(4):1197-215.

2. Cuerda C, Schneider S, Van Gossum A. Clinical nutrition education in medical schools: results of an Espen survey. Clin Nutr. 2017;36(4):915-6.

3. Pôrto PA, Mendonça SS. Conhecimento dos profissionais de enfermagem e médicos de um hospital público sobre terapia nutricional. Rev Bras Nutr Clín. 2015;30(3):227-34.

4. Cunha HF, Rocha EEM, Hissa M. Práticas em terapia nutricional entre médicos: inquérito via internet. Rev. Bras Nutr Clín. 2014;29(4):335-41.

5. Massimi M. Alimentos, palavras e saúde (da alma e do corpo), em sermões de pregadores brasileiros do século XVII. Hist Ciênc SaúdeManguinhos. 2006;13(2):253-70.

6. Raman M, Violato C, Coderre S. How much do gastroenterology fellows know about nutrition? J Clin Gastroenterol. 2009;43(6):559-64.

7. Crawford AL, Aspry KE. Teaching doctors-in-training about nutrition: where are we going in 2016? R I Med J. 2016;99:23-5.

8. Kris-Etherton P, Akabas S, Douglas P, Kohlmeier M, Laur C, Lenders $\mathrm{C}$, et al. Nutrition competencies in health professionals' education and training: a new paradigm. Adv Nutr. 2015;6(1):83-7. 
9. Hark L, Deen D. Position of the Academy of Nutrition and Dietetics: interprofessional education in nutrition as an essential component of medical education. Journal of the Academy of Nutrition and Dietetics. 2017;117(7):1104-13.

10. Castro M, Pompilio C, Horie L, Verotti C, Waitzberg D. Education program on medical nutrition and length of stay of critically ill patients. Clin Nutr. 2013;32(6):1061-66.

11. Kris-Etherton P, Akabas S, Bales C, Bistrian B, Braun L, Edwards $\mathrm{M}$, et al. The need to advance nutrition education in the training of health care professionals and recommended research to evaluate implementation and effectiveness. Am J Clin Nutr. 2014;99(5):1153S-1166S.

12. Borghi R, Meale MM, Gouveia MAP, França JID, Damião AOMC. Perfil nutricional de pacientes internados no Brasil: análise de 19.222 pacientes (Estudo BRAINS). Rev Bras Nutr Clín. 2013;28(4):255-63.

13. Bottoni A, Hassan DZ, Nacarato A, Garnes SA, Bottoni A. Porque se preocupar com a desnutrição hospitalar?: revisão de literatura. J Health Sci Inst. 2014;32(3):314-7.

14. Han S, Auer R, Cornuz J, Marques-Vidal P. Clinical nutrition in primary care: an evaluation of resident physicians' attitudes and selfperceived proficiency. Clin Nutr ESPEN. 2016;15:69-74.

15. Walsh C, Ziniel S, Delichatsios H, Ludwig D. Nutrition attitudes and knowledge in medical students after completion of an integrated nutrition curriculum compared to a dedicated nutrition curriculum: a quasi-experimental study. BMC Med Educ. 2011;11(1):1-7.

16. Ray S, Ball L, Crowley J, Laur C, Rajput-Ray M, Gillam S. Nutrition in medical education: reflections from an initiative at the University of Cambridge. Journal of Multidisciplinary Healthcare. 2014;7:209-215.

17. Mogre V, Stevens F, Aryee P, Amalba A, Scherpbier A. Why nutrition education is inadequate in the medical curriculum: a qualitative study of students' perspectives on barriers and strategies. BMC Med Educ. 2018;18(1):1-11

18. Devries S, Agatston A, Aggarwal M, Aspry K, Esselstyn C, KrisEtherton $\mathrm{P}$, et al. A deficiency of nutrition education and practice in cardiology. Am J Med. 2017;130(11):1298-305.

19. Singh H, Duerksen D. Survey of clinical nutrition practices of Canadian gastroenterologists. Can J Gastroenterol. 2006;20(8):527-30.

20. Ball L, Barnes K, Laur C, Crowley J, Ray S. Setting priorities for research in medical nutrition education: an international approach. BMJ Open. 2016;6(12):e013241.

21. Sacks G. The shrinking of formalized nutrition education in health professions curricula and postgraduate training. JPEN J Parenter Enteral Nutr. 2017;41(2):217-25.

22. Adams K, Kohlmeier M, Zeisel S. Nutrition education in U.S. medical schools: latest update of a national survey. Acad Med. 2010;85(9):1537-42.

23. Kiraly L, McClave S, Neel D, Evans D, Martindale R, Hurt R. Physician nutrition education. Nutrition in Clinical Practice. 2014;29(3):332-7.

24. Ferreira HCC, Rodrigues PA. Avaliação do conhecimento médico sobre terapia nutricional parenteral em um hospital público do Distrito Federal. BRASPEN J. 2017;32(4):387-393.

25. Pazin-Filho A, Frezza G, Matsuno A, Alcântara S, Cassiolato S, Bitar J, et al. Princípios de prescrição médica hospitalar para estudantes de medicina. Medicina (Ribeirao Preto, Online). 2013;46(1):183-194
26. Orimo $\mathrm{H}$, Ueno $\mathrm{T}$, Yoshida $\mathrm{H}$, Sone $\mathrm{H}$, Tnaka A, Itakura $\mathrm{H}$. Nutrition education in japanese medical schools: a follow-up survey. Asia Pac J Clin Nutr. 2013;22(1):144-149

27. Sodjinou R, Bosu WK, Fanou N, Déart L, Kupka R, Tchibindat F et al. Nutrition training in medical and other health professional schools in West Africa: the need to improve current approaches and enhance training effectiveness. Golb Health Action. 2014;7:24827-35

28. Danek RL, Berlin KL, Waite GN, Geib RW. Perceptions of nutrition education in the current medical school curriculum. Fam Med. 2017;49(10):803-806

29. Cuerda C, Muscaritoli M, Donini LM, Schneider S, Vargas JA, Gossum AV et al. Nutrition education in medical schools (NEMS). An ESPEN position paper. Clin Nutr. 2019;38(3):969-974

30. Spear S, Sim V, Moore F, Todd S. Just say no to intensive care unit starvation. Nutrition in Clinical Practice. 2013;28(3):387-91.

31. Waitzberg DL1, Caiaffa WT, Correia MI. Hospital malnutrition: the Brazilian national survey (IBRANUTRI): a study of 4000 patients. Nutrition. 2001 Jul-Aug;17(7-8):573-80.

32. Toledo DO, Piovacari SMF, Horie LM, Matos LBN, Castro MG, Ceniccola GD, et al. Campanha "Diga não à desnutrição": 11 passos importantes para combater a desnutrição hospitalar. Braspen J. 2018;33(1):86-100.

33. Agência Senado. Sancionada lei que obriga escolas a incluir educação alimentar e nutricional no currículo acadêmico. Brasília, 2019 [acesso 20 ago 2019]. Disponível em: https://www12.senado.leg. br/noticias/audios/2018/05/sancionada-lei-que-obriga-escolas-aincluir-educacao-alimentar-e-nutricional-no-curriculo-academico.

34. Baute V, Carr A, Blackwell J, Carstensen E, Chhabra P, Porter, L et al. Incorporating formal nutrition education into a medical school curriculum: a student-initiated lecture series. The Am J Med. 2017;130(6):623-5

35. Perlstein R, McCoombe S, Shaw C, Nowson C. Medical students perceptions regarding the importance of nutritional knowledge and their confidence in providing competent nutrition practice. Public Health. 2016;140:27-34.

36. United States of America. National Research Council (US) Committee on Nutrition in Medical Education. Nutrition Education in U.S. Medical Schools. Washington, DC: National Academy Press; 1985.

37. Laur C, Ball L, Ahankari A, Avdagovska M, Crowley J, Deen D, et al. Proceedings of the inaugural International Summit for Medical Nutrition Education and Research. Public Health. 2016;140:59-67.

38. Associação Brasileira para Estudo da Obesidade e da Síndrome Metabólica. 2019 [acesso 14 jun 2019]. Disponível em: http://www. abeso.org.br/atitude-saudavel/mapa- obesidade.

39. Correia MITD, Perman MI, Waitzberg DL. Hospital malnutrition in Latin America: a systematic review. Clin Nutr (Edinb.). 2017;36( 4):958-67.

40. Quaresma EM, Villacorta DBV, Silva RA, Veríssimo AOL, Caldato MCF. Análise de compreensão a respeito da terapia nutricional em unidades de terapia intensiva de um hospital escola. Revista Eletrônica Acervo Saúde. 2019;9(3):1112-7.

41. Schlair S, Hanley K, Gillespie C, Disney L, Kalet A, Darby P, et al. How medical students' behaviors and attitudes affect the impact of a brief curriculum on nutrition counseling. J Nutr Educ Behav.

REVISTA BRASILEIRA DE EDUCAÇÃO MÉDICA

9 $94(4): \mathrm{e} 107 ; 2020$ 


$$
\text { 2012;44(6):653-7. }
$$

42. Afaghi A, Haj Agha Mohamadi A, Ziaee A, Sarchami R. Effect of an integrated case-based nutrition curriculum on medical education at Qazvin University of Medical Sciences, Iran. Global Journal of Health Science. 2011;4(1):112-117

43. Crowley J, Ball L, Leveritt MD, Arroll B, Han DY, Wall C. Impact of an undergraduate course on medical students' self-perceived nutrition intake and self-efficacy to improve their health behaviours and counselling practices. J Prim Health Care. 2014 Jun 1;6(2):101-7.

44. Brasil. Ministério da Educação. Diretrizes Curriculares Nacionais do Curso de Graduação em Medicina. Brasília; 2014 [acesso em 22 jul 2019]. Disponível em: http://portal.mec.gov.br/index. php?option=com_docman\&view=download\&alias=15874-rces00314\&category_slug=junho-2014-pdf\&Itemid=30192.

45. Neumann C, Gerbase M, Blank D, Capp E, Oliveira M. Avaliação de competências no internato: atividades profissionais confiabilizadoras essenciais para a prática médica. Porto Alegre: UFCSPA, UFRGS; 2019.

46. Frantz D, McClave S, Hurt R, Miller K, Martindale R. Cross-sectional study of U.S. interns' perceptions of clinical nutrition education. JPEN J Parenter Enteral Nutr. 2016;40(4):529-35.

47. Kushner R, Van Horn L, Rock C, Edwards M, Bales C, Kohlmeier M, et al. Nutrition education in medical school: a time of opportunity. Am J Clin Nutr. 2014;99(5):1167S-1173S.

48. Brasil. Ministério da Saúde. Secretaria de Vigilância em Saúde. Departamento de Vigilância de Doenças e Agravos nas Transmissíveis e Promoção de Saúde. Vigitel Brasil 2016: vigilância de fatores de risco e proteção para doenças crônicas por inquérito telefônico: estimativas sobre frequência e distribuição sociodemográfica de fatores de risco e proteção para doenças crônicas nas capitais dos 26 estados brasileiros e no Distrito Federal em 2016. Brasília: Ministério da Saúde; 2017.

49. Bastos L. OPAS/OMS Brasil - Doenças cardiovasculares. Pan American Health Organization, World Health Organization; 2019 [acesso em 15 jun 2019]. Disponível em: https://www.paho.org/bra/ index.php?option=com_content $\&$ view $=$ article $\&$ id $=5253:$ doencas cardiovasculares\&Itemid $=1096$

50. Instituto Nacional do Câncer. Ministério da Saúde; 2019 [Acesso em 28 de Jul 2019] Disponível em: https://www.inca.gov.br/mitos-everdades/alimentacao.

51. Brasil. Resolução da Diretoria Colegiada no 63, 2000. Brasília; 2000. [Acesso em 15 Jul 2019] Disponível em: https://www20.anvisa.gov. br/segurancadopaciente/index.php/legislacao/item/resolucao-dadiretoria-colegiada-rcd-n-63-de-6-de-julho-de-2000.

52. Brasil. Portaria no 272, 1998. Brasília; 1998. [Acesso em 10 Jul 2019] Disponível em: http://bvsms.saude.gov.br/bvs/saudelegis/svs1/1998/ prt0272_08_04_1998.html.

\section{CONTRIBUIÇÃO DOS AUTORES}

Denise Bitar Vasconcelos Villacorta participou da pesquisa em bases de dados, da coleta de resultados, da escrita do artigo, da revisão dos resultados apresentados e da revisão geral do manuscrito. Charles Alberto Villacorta de Barros participou da pesquisa em bases de dados, da coleta de resultados, da escrita do artigo, da revisão dos resultados apresentados e da revisão geral do manuscrito. Bernardo Felipe Santana de Macedo participou da revisão textual, da formatação gráfica e da finalização para publicação. Milena Coelho Fernandes Caldato foi responsável pela orientação do trabalho, participou da coleta dos resultados, e revisou periodicamente a progressão do artigo.

\section{CONFLITO DE INTERESSES}

Os autores declaram não haver conflito de interesses neste estudo.

\section{ENDEREÇO PARA CORRESPONDÊNCIA}

Denise Bitar Vasconcelos Villacorta. Rua Domingos Marreiros, 907, ap. 1402, Umarizal, Belém, PA, Brasil. CEP: 66055-215.

E-mail: denibvv@gmail.com 